\title{
Bis(alkyl)thioethers on a biphenyl scaffold: A spectroscopic and structural insight
}

Rhiann Ferguson ${ }^{a}$, Phillip S. Nejman ${ }^{a}$, Alexandra M. Z. Slawin ${ }^{a}$ and J. Derek Woollins ${ }^{a, *}$

a EaStCHEM School of Chemistry, University of St Andrews, St Andrews, Fife, KY16 9ST, Scotland

* Corresponding author: E-mail: jdw3@st-and.ac.uk

\begin{abstract}
A series of bis(alkyl)thioether compounds utilising biphenyl as the backbone of the type 2,2'bis(alkylthio)-1,1'-biphenyl have been prepared with yields of $32-70 \%$. The six benzyl derivatives all displayed an $A B$ quartet for the $\mathrm{CH}_{2}$ hydrogens within their ${ }^{1} \mathrm{H}$ NMR spectra due to restricted rotation within the molecule. Where the alkyl group was changed to neopentyl the $\mathrm{CH}_{2}$ protons became equivalent giving rise to a singlet within the ${ }^{1} \mathrm{H}$ NMR spectrum. The compounds have been characterised principally using multinuclear NMR spectroscopy and single crystal X-ray diffraction.
\end{abstract}

\section{Keywords}

Thioether, sulfur; biphenyl; NMR spectroscopy; X-Ray structure 


\section{Introduction}

Organosulfur compounds have a long and rich history in organic synthesis acting as valuable intermediates. $[1,2]$ Compounds containing $C-S$ bonds are highly desirable, in particular alkyl aryl and dialkyl sulfides, resulting in a large number of reported methods for their synthesis.[3] Thioethers have a wide and varied use within chemistry with compounds containing this sulfur linkage studied in medicinal chemistry (A),[4] as ligands in organometallic chemistry (B) [5] and in catalysis (C) (Figure 1).[6] Additionally, compounds of this type have been shown to be useful precursors for the formation of other highly interesting species.[7]<smiles>Cn1nccc1-c1ccc(Sc2cccc(C3(C(N)=O)CCOCC3)c2)cc1</smiles>

A<smiles>[R1]C1CSCCSCCSC1</smiles>

$\mathrm{R}=\mathrm{H}, \mathrm{Me}$

B<smiles>[R][SH]1(C)(I)c2ccccc2N2CCN([Al])C21</smiles>

$\mathrm{R}={ }^{i} \mathrm{Pr}, \mathrm{Me}, \mathrm{Ph}$

C

Figure 1: Compounds demonstrating the various uses of thioethers.

Of particular relevance to the work presented here are bis(alkyl)thioethers, and their derivatives, attached to polyaromatic backbones such as acenaphthene, naphthalene, biphenyl and binaphthalene. The chemistry of these systems has seen fair less investigation than those based upon the 1,2-benzene scaffold. A number of reports have been published describing the synthesis of simple thioether compounds (D), $[8-11](\mathbf{E})[12,13]$ and (F)[14] (Figure 2). However, to date there has been no report of the acenaphthene derivative. The work conducted by Barbero, which describes the synthesis of $\mathbf{E}$, was examining synthetic routes to arene mono- and disulfonyl chlorides which are key intermediates for the synthesis of strong Brønsted acids which are useful organocatalysts.[13] Longer chain alkyl groups have also been utilised including ethyl derivatives of naphthalene (G)[15] and biphenyl (H)[12] as well as a butyl analogue on naphthalene (I)[16] (Figure 2). 
<smiles>CSc1cccc2cccc(SC)c12</smiles>

D<smiles>CCSc1cccc2cccc(SCC)c12</smiles>

G<smiles>CSc1ccccc1-c1ccccc1SC</smiles>

E<smiles>CCSc1ccccc1-c1ccccc1SCC</smiles>

H<smiles>CSc1ccc2ccccc2c1-c1c(SC)ccc2ccccc12</smiles>

$\mathbf{F}$<smiles>CCCCSc1cccc2cccc(SCCCC)c12</smiles>

I

Figure 2: Simple thioether compounds based upon polyaromatic backbones.

Thioethers bound to polyaromatic backbones are not limited to only alkyl derivatives. A variety of bis(aryl)thioethers and their derivatives attached to naphthalene, acenaphthene, biphenyl and binaphthalene are also known. Figure 3 (J-M) shows the phenyl analogues that have been prepared based upon these backbones.[15, 17-19] As part of a wider investigation into using peri-substitution in the formation of novel bonding motifs Aschenbach and Knight prepared compounds $\mathbf{K}$ and $\mathbf{J}$ respectively alongside other chalcogen derivatives including selenium and tellurium analogues. In both cases the onset of an attractive interaction, in the form of a 3c-4e bond, across the peri-gap was proposed based upon single crystal X-ray diffraction data and computational analysis (B3LYP level of DFT).[15, 17] A one-pot synthesis of 2,2'-bis-substituted biphenyls, of which $\mathbf{L}$ is an example, was developed by Studer in 2015. This involved the addition of Mg-thiolates to benzyne followed by homocoupling of the resulting ortho-substituted arylmagnesium species. They found a number of the 2,2'-bis-substituted biphenyls readily crystallised revealing interesting interactions within the solid state as determined by single crystal X-ray diffraction.[18] 


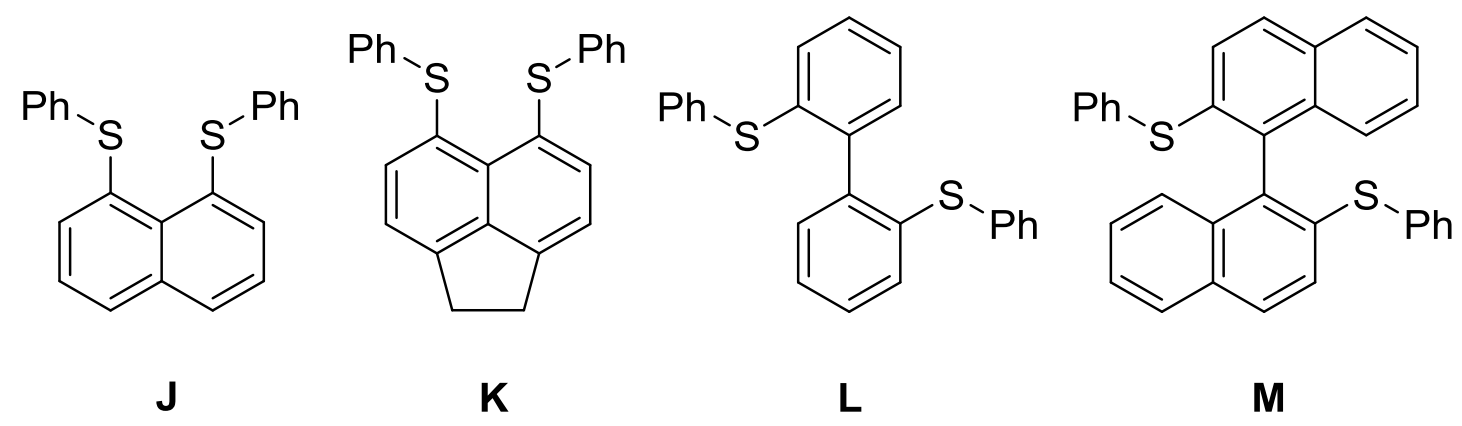

Figure 3: bis(aryl)thioether compounds on polyaromatic backbones.

The Woollins group has recently been investigating the use of rigid organic backbones such as naphthalene and acenaphthene in the formation of novel bonding interactions (3c-4e bonds) between chalcogens.[15, 17, 20-22] Building on this work we proposed to introduce a more rotationally free backbone to investigate the importance of the rigidity on the potential formation of a $3 c-4$ e bond. Biphenyl was selected as the backbone as a number of potential synthetic routes to the desired chalcogenoether compounds could be designed based upon the known literature compounds 2,2'-dibromo-1,1'-biphenyl (1) and dibenzo[c,e][1,2]dithiine (2). Additionally, the introduction of axially chirality from the biphenyl backbone makes these compounds of interest as potential ligands for asymmetric catalysis[23] as they represent a simpler alternative to, for example, binaphthalene. The work presented here represents the first step in designing synthetic routes towards the related selenium and tellurium chalcogenoethers. In this work we describe the synthesis and crystal structures of six 2,2'-bis(benzylthio)-1,1'-biphenyl compounds with varying para substituents on the benzyl group. Additionally 2,2'-bis(neopentylthio)-1,1'-biphenyl has been prepared and spectroscopically characterised. The features of their ${ }^{1} \mathrm{H}$ NMR spectra and solid state structures are examined and compared.

\section{Synthetic Methods}

\subsection{General}

All synthetic manipulations were performed under an atmosphere of dry nitrogen using standard Schlenk-line techniques. Dry solvents were either collected from an MBraun Solvent Purification System, or were dried and stored according to common procedures.[24] Chemicals were purchased from Sigma Aldrich, Acros Organics, Alfa Aesar or were taken from the laboratory inventory and used without further purification. 2,2'-dibromo-1,1'-biphenyl was prepared according to the literature procedure.[25] Dibenzo[c,e][1,2]dithiine and 1,1'-biphenyl-2,2'-dithiol were synthesised according to modified literature procedures.[26, 27] The synthetic protocols for 1-3 are included within the supplementary information for completeness. All NMR spectra were recorded using a 
Bruker Avance II 400 or Bruker Avance III 500 spectrometer at $25{ }^{\circ} \mathrm{C}$. Assignments of ${ }^{1} \mathrm{H}$ and ${ }^{13} \mathrm{C} \mathrm{NMR}$ spectra were made with the use of $\mathrm{H}-\mathrm{H}$ DQF-COSY, $\mathrm{H}-\mathrm{C}$ HSQC, $\mathrm{H}-\mathrm{C}$ HMBC two-dimensional experiments. For ${ }^{1} \mathrm{H}$ and ${ }^{13} \mathrm{C}$ NMR tetramethylsilane was used as an external standard, for ${ }^{19} \mathrm{~F}\left\{{ }^{1} \mathrm{H}\right\}$ $\mathrm{NMR} \mathrm{CCl}_{3} \mathrm{~F}$ was used as an external standard. Residual solvent peaks were also used for calibration $\left(\mathrm{CDCl}_{3} \delta_{\mathrm{H}} 7.26, \delta_{\mathrm{C}} 77.2 \mathrm{ppm}\right)$. Chemical shifts $(\delta)$ are given in parts per million (ppm). Coupling constants $(J)$ are given in Hertz $(\mathrm{Hz})$. Mass Spectrometry spectra were acquired at the EPSRC UK National Mass Spectrometry Facility in Swansea using a Waters Xevo G2-S (ASAP). Infrared spectra were recorded as $\mathrm{KBr}$ discs in the range of $4000-400 \mathrm{~cm}^{-1}$ using a Perkin-Elmer System 2000 NIR Fourier Transform spectrometer. Elemental analyses were performed by Stephen Boyer at the London Metropolitan University.

\subsection{Experimental}

\section{General Method A}

To a solution of 3 (1 eq.) dissolved in ethanol ( $15 \mathrm{~mL}$ ), sodium hydroxide (2.2 eq.) was added and the solution stirred for 30 minutes. The benzyl bromide ( 2.2 eq.) was added to the reaction flask, and the resulting mixture stirred for 24 hours at room temperature. The solvent was removed and the crude product dissolved in diethyl ether $(20 \mathrm{~mL})$. The suspension was filtered under vacuum to remove the salt by-product. The filtrate was concentrated under reduced pressure yielding the crude product. Recrystallisation from ethanol afforded the product.

\section{2,2'-bis(benzylthio)-1,1'-biphenyl (4)}

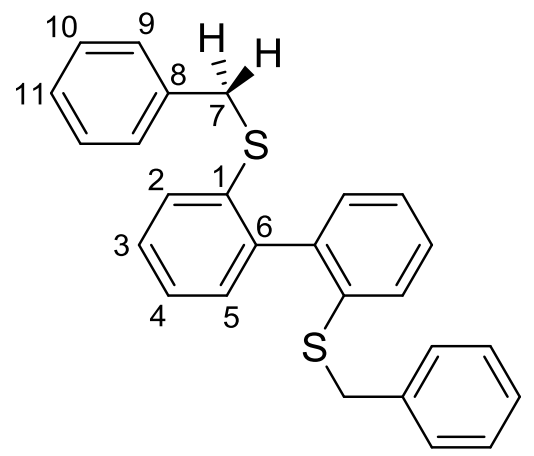

NMR atom numbering for 4 .

Prepared following method A using 3 (100 mg, $0.46 \mathrm{mmol}$ ), sodium hydroxide ( $39 \mathrm{mg}, 1.01 \mathrm{mmol}$ ) and benzyl bromide $(0.12 \mathrm{~mL}, 1.01 \mathrm{mmol})$. Compound 4 was obtained as a white crystalline solid (96 $\mathrm{mg}, 0.24 \mathrm{mmol}, 53 \%$ ), m.p $110-113^{\circ} \mathrm{C}$ (lit. $116^{\circ} \mathrm{C}$ ).[12] Crystals suitable for X-ray work were obtained by recrystallisation from ethanol. Anal. calcd. for $\mathrm{C}_{26} \mathrm{H}_{22} \mathrm{~S}_{2}\left(398.58 \mathrm{~g} \mathrm{~mol}^{-1}\right): \mathrm{C}, 78.34 ; \mathrm{H}, 5.56$. Found 
C, 78.27; $\mathrm{H}, 5.66 .{ }^{1} \mathrm{H}$ NMR: $\delta_{\mathrm{H}}\left(400.1 \mathrm{MHz}, \mathrm{CDCl}_{3}\right) 7.37\left(2 \mathrm{H}, \mathrm{dd},{ }^{3} \int_{\mathrm{HH}} 7.9,{ }^{4} J_{\mathrm{HH}} 1.3 \mathrm{~Hz}, \mathrm{H}-5\right), 7.29(2 \mathrm{H}$, pseudo-td, $\left.{ }^{3} J_{\mathrm{HH}} 7.6,{ }^{4} J_{\mathrm{HH}} 1.6 \mathrm{~Hz}, \mathrm{H}-4\right), 7.24-7.16(12 \mathrm{H}, \mathrm{m}, \mathrm{H}-3,9,10,11), 7.07\left(2 \mathrm{H}, \mathrm{dd},{ }^{3} \int_{\mathrm{HH}} 7.5,1.6 \mathrm{~Hz}\right.$, $\mathrm{H}-2), 3.99\left(4 \mathrm{H}, \mathrm{ABq}, \Delta \delta_{\mathrm{AB}} 0.02,{ }^{2} \jmath_{\mathrm{HH}} 12.9 \mathrm{~Hz}, \mathrm{H}-7\right) .{ }^{13} \mathrm{C}$ NMR: $\delta_{\mathrm{c}}\left(100.6 \mathrm{MHz}, \mathrm{CDCl}_{3}\right) 141.1\left(\mathrm{ArC}_{q}, \mathrm{C}-6\right)$, 137.3 ( $\left.\mathrm{ArC}_{\mathrm{q}}, \mathrm{C}-8\right), 136.3\left(\mathrm{ArC}_{\mathrm{q}}, \mathrm{C}-1\right), 130.5$ (ArCH, C-5), 129.1 (ArCH, C-9), 128.9 (ArCH,C-2), 128.5 (ArCH, C-10), 128.3 (ArCH, C-3), 127.2 (ArCH, C-11), 125.9 (ArCH, C-4), $38.6\left(\mathrm{CH}_{2}, \mathrm{C}-7\right)$. HRMS (APCl+): $m / z(\%) 399.1236(20)[\mathrm{M}+\mathrm{H}]^{+}, 321.0767(5)\left[\mathrm{M}-\mathrm{C}_{6} \mathrm{H}_{5}\right]^{+}, 307.0610(10)\left[\mathrm{M}-\mathrm{C}_{7} \mathrm{H}_{7}\right]^{+}, 287.0889$ (15) $\left[\mathrm{M}-\mathrm{C}_{7} \mathrm{H}_{8} \mathrm{~S}\right]^{+}, 229.0140(25)\left[\mathrm{M}-\mathrm{C}_{13} \mathrm{H}_{13}\right]^{+}, 181.1011$ (100). IR (KBr): $\mathrm{v}_{\max } / \mathrm{cm}^{-1} 3126 \mathrm{w}\left(\mathrm{v}_{\mathrm{Ar}-\mathrm{H}}\right), 2922 \mathrm{~m}\left(\mathrm{v}_{\mathrm{C}-}\right.$ H), 1578m, 1510m, 1424m, 1041m, 1069s, 717s ( $\left.v_{\text {C-s }}\right)$. Raman (glass capillary): $v_{\max } / \mathrm{cm}^{-1} 3053 \mathrm{~s}\left(\mathrm{~V}_{\mathrm{Ar}-\mathrm{H}}\right)$, $2924 \mathrm{~m}\left(\mathrm{v}_{\mathrm{C}-\mathrm{H}}\right), 1588 \mathrm{~s}, 1237 \mathrm{~m}, 1040 \mathrm{~m}, 1003 \mathrm{~s}, 723 \mathrm{~s}\left(\mathrm{v}_{\mathrm{C}-\mathrm{s}}\right), 466 \mathrm{~m}$.

\section{2,2'-bis((4-methylbenzyl)thio)-1,1'-biphenyl (5)}

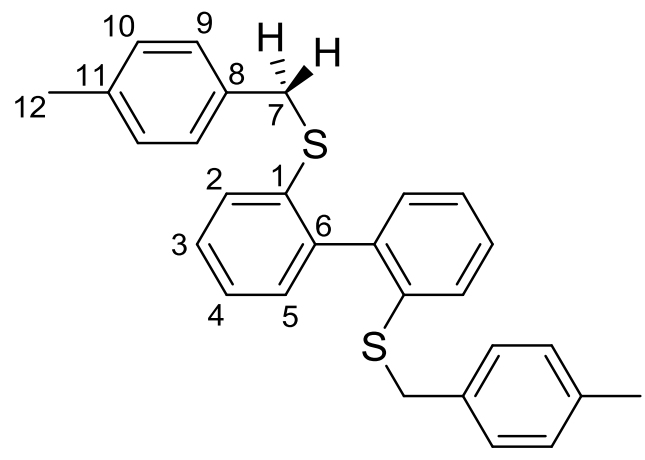

NMR atom numbering for 5.

Prepared following method A using 3 (118 $\mathrm{mg}, 0.54 \mathrm{mmol}$ ), sodium hydroxide (46 mg, $1.18 \mathrm{mmol}$ ) and 4-methylbenzyl bromide ( $220 \mathrm{mg}, 1.18 \mathrm{mmol}$ ). Compound 5 was obtained as a white crystalline solid (103 mg, $0.24 \mathrm{mmol}, 45 \%$ ), m.p $128-130{ }^{\circ} \mathrm{C}$. Crystals suitable for X-ray work were obtained by recrystallisation from ethanol. Anal. calcd. for $\mathrm{C}_{28} \mathrm{H}_{26} \mathrm{~S}_{2}\left(426.63 \mathrm{~g} \mathrm{~mol}^{-1}\right): \mathrm{C}, 78.83 ; \mathrm{H}, 6.14$. Found $\mathrm{C}$, 78.69; $\mathrm{H}, 6.24 .{ }^{1} \mathrm{H}$ NMR: $\delta_{\mathrm{H}}\left(500.1 \mathrm{MHz}, \mathrm{CDCl}_{3}\right) 7.38\left(2 \mathrm{H}, \mathrm{dd},{ }^{3} \mathrm{~J}_{\mathrm{HH}} 7.9,{ }^{4} \mathrm{~J}_{\mathrm{HH}} 1.3 \mathrm{~Hz}, \mathrm{H}-2\right) 7.30(2 \mathrm{H}$, pseudo-td, $\left.{ }^{3} J_{\mathrm{HH}} 7.6,{ }^{4} J_{\mathrm{HH}} 1.6 \mathrm{~Hz}, \mathrm{H}-3\right), 7.21\left(2 \mathrm{H}\right.$, pseudo-td, $\left.{ }^{3} J_{\mathrm{HH}} 7.4,{ }^{4} J_{\mathrm{HH}} 1.3 \mathrm{~Hz}, \mathrm{H}-4\right), 7.14-7.08(6 \mathrm{H}, \mathrm{m}$, $\mathrm{H}-5,9), 7.04\left(4 \mathrm{H}, \mathrm{d},{ }^{3} J_{\mathrm{HH}} 7.9 \mathrm{~Hz}, \mathrm{H}-10\right), 3.98\left(4 \mathrm{H}, \mathrm{ABq}, \Delta \delta_{\mathrm{AB}} 0.02,{ }^{2} J_{\mathrm{HH}} 12.8 \mathrm{~Hz}, \mathrm{H}-7\right), 2.31(6 \mathrm{H}, \mathrm{s}, \mathrm{H}-12)$. ${ }^{13} \mathrm{C}$ NMR: $\delta_{\mathrm{c}}\left(125.8 \mathrm{MHz}, \mathrm{CDCl}_{3}\right) 140.9\left(\mathrm{ArC}_{q}, \mathrm{C}-6\right), 136.8\left(\mathrm{ArC}_{\mathrm{q}}, \mathrm{C}-11\right), 136.6\left(\mathrm{ArC}_{\mathrm{q}}, \mathrm{C}-1\right), 134.1\left(\operatorname{ArC}_{\mathrm{q}}, \mathrm{C}-\right.$ 8), 130.5 ( $\mathrm{ArCH}, \mathrm{C}-5), 129.2$ ( $\mathrm{ArCH} \mathrm{C}-10)$,128.9 ( $\mathrm{ArCH}, \mathrm{C}-9), 128.7$ ( $\mathrm{ArCH}, \mathrm{C}-2), 128.3(\mathrm{ArCH}, \mathrm{C}-3)$, 125.7 (ArCH, C-4), $38.1\left(\mathrm{CH}_{2}, \mathrm{C}-7\right), 21.2\left(\mathrm{CH}_{3}, \mathrm{C}-12\right)$. HRMS (APCl+): $m / z$ (\%) 427.1539 (3) [M+H] $]^{+}$, $105.0693(100)\left[\mathrm{M}-\mathrm{C}_{20} \mathrm{H}_{17} \mathrm{~S}_{2}\right]^{+}$. IR ( $\left.\mathrm{KBr}\right): \mathrm{v}_{\max } / \mathrm{cm}^{-1} 3052 \mathrm{w}\left(\mathrm{v}_{\mathrm{Ar}-\mathrm{H}}\right), 2919 \mathrm{~m}\left(\mathrm{v}_{\mathrm{c}-\mathrm{H}}\right), 1579 \mathrm{~s}, 1452 \mathrm{~s}, 1087 \mathrm{~m}$, 1040s, $741 \mathrm{~m}$ ( $\left.v_{\text {C-s }}\right)$. Raman (glass capillary): $v_{\max } / \mathrm{cm}^{-1} 3060 \mathrm{~s}\left(\mathrm{v}_{\text {Ar-H}}\right), 2922 \mathrm{~m}\left(\mathrm{v}_{\mathrm{C}-\mathrm{H}}\right), 1587 \mathrm{~m}, 1237 \mathrm{~m}$, $1042 \mathrm{~m}, 744 \mathrm{~s}\left(\mathrm{v}_{\mathrm{c}-\mathrm{s}}\right)$. 


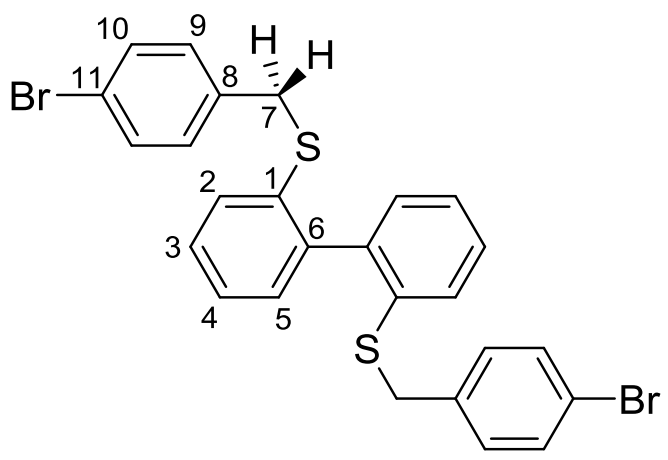

NMR atom numbering for 6 .

Prepared following method A using 3 (104 mg, $0.48 \mathrm{mmol}$ ), sodium hydroxide (40 mg, $1.05 \mathrm{mmol}$ ) and 4-bromobenzyl bromide $(260 \mathrm{mg}, 1.05 \mathrm{mmol})$. Compound 6 was obtained as white needle like crystals (132 mg, $0.24 \mathrm{mmol}, 51 \%$ ), m.p $125-127^{\circ} \mathrm{C}$. Crystals suitable for X-ray work were obtained by recrystallisation from ethanol. Anal. calcd. for $\mathrm{C}_{26} \mathrm{H}_{20} \mathrm{Br}_{2} \mathrm{~S}_{2}\left(556.37 \mathrm{~g} \mathrm{~mol}^{-1}\right)$ : C, 56.12; $\mathrm{H}, 3.62$. Found C, 55.98; $\mathrm{H}, 3.60 .{ }^{1} \mathrm{H}$ NMR: $\delta_{\mathrm{H}}\left(500.1 \mathrm{MHz}, \mathrm{CDCl}_{3}\right)$ 7.38-7.30 (8H, m, H-2, 3, 10), $7.25(2 \mathrm{H}$, pseudo-td, $\left.{ }^{3} \mathrm{HH}_{\mathrm{HH}} 7.4,{ }^{4} \mathrm{~J}_{\mathrm{HH}} 1.2 \mathrm{~Hz}, \mathrm{H}-4\right), 7.06\left(2 \mathrm{H}, \mathrm{dd},{ }^{3} \mathrm{~J}_{\mathrm{HH}} 7.5,{ }^{4} \mathrm{~J}_{\mathrm{HH}} 1.4 \mathrm{~Hz}, \mathrm{H}-5\right), 7.04\left(4 \mathrm{H}, \mathrm{d},{ }^{3} \mathrm{~J}_{\mathrm{HH}} 8.3, \mathrm{H}-9\right)$, $3.87\left(4 \mathrm{H}, \mathrm{ABq}, \Delta \delta_{\mathrm{AB}} 0.02,{ }^{2} J_{\mathrm{HH}} 12.9 \mathrm{~Hz}, \mathrm{H}-7\right) .{ }^{13} \mathrm{C} N M R: \delta_{\mathrm{c}}\left(125.8 \mathrm{MHz}, \mathrm{CDCl}_{3}\right) 141.3\left(\mathrm{ArC}_{q}, \mathrm{C}-6\right), 136.3$ $\left(\mathrm{ArC}_{\mathrm{q}}, \mathrm{C}-8\right), 135.4$ ( $\left.\mathrm{ArC}_{\mathrm{q}}, \mathrm{C}-1\right), 131.4$ (ArCH, C-10), 130.5 (ArCH, C-9), 130.4 (ArCH, C-5), 129.4 (ArCH, C-2), 128.3 ( $\mathrm{ArCH}, \mathrm{C}-3), 126.2$ (ArCH, C-4), 120.9 ( $\left.\mathrm{ArC}_{\mathrm{q}}, \mathrm{C}-11\right), 37.9\left(\mathrm{CH}_{2}, \mathrm{C}-7\right)$. HRMS (APCl+): m/z (\%) $184.0334(15)\left[\mathrm{M}-\mathrm{C}_{14} \mathrm{H}_{12} \mathrm{Br}{ }_{2} \mathrm{~S}\right]^{+}, 170.9620\left({ }^{81} \mathrm{Br}\right) \& 168.9640\left({ }^{79} \mathrm{Br}\right)(100)\left[\mathrm{M}-\mathrm{C}_{19} \mathrm{H}_{14} \mathrm{BrS}\right]^{+}$. IR $(\mathrm{KBr}):$ $v_{\max } / \mathrm{cm}^{-1} 3054 \mathrm{w}\left(\mathrm{v}_{\mathrm{Ar}-\mathrm{H}}\right), 1560 \mathrm{~m}, 1454 \mathrm{~s}, 1423 \mathrm{~s}, 1214 \mathrm{~s}, 1025 \mathrm{~m}, 760 \mathrm{~s}\left(\mathrm{v}_{\mathrm{C}-\mathrm{Br}}\right), 723 \mathrm{~m}\left(\mathrm{v}_{\mathrm{C}-\mathrm{s}}\right)$. Raman (glass capillary): $v_{\max } / \mathrm{cm}^{-1} 3047 \mathrm{~s}\left(\mathrm{v}_{\mathrm{Ar}-\mathrm{H}}\right), 2923 \mathrm{w}\left(\mathrm{v}_{\mathrm{C}-\mathrm{H}}\right), 1588 \mathrm{~s}, 1485 \mathrm{~s}, 1069 \mathrm{~m}, 750\left(\mathrm{v}_{\mathrm{C}-\mathrm{Br}}\right)$. 


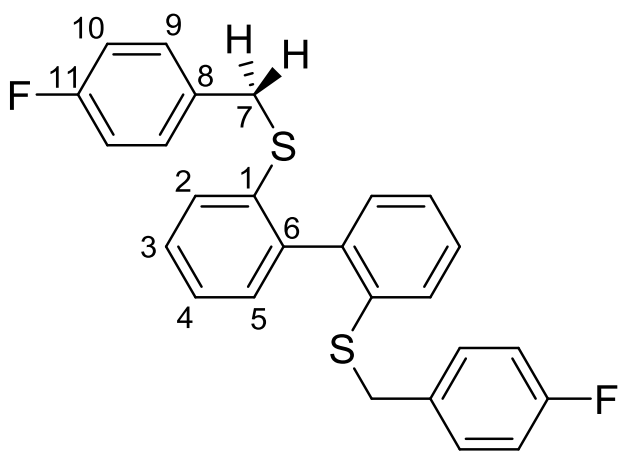

NMR atom numbering for 7 .

Prepared following method A using 3 (108 mg, $0.50 \mathrm{mmol}$ ), sodium hydroxide ( $39 \mathrm{mg}, 1.0 \mathrm{mmol}$ ) and 4-fluorobenzyl bromide $(0.12 \mathrm{~mL}, 1.0 \mathrm{mmol})$. Compound 7 was obtained as white needle crystals (148 mg, $0.34 \mathrm{mmol}, 69 \%$ ), m.p $108-110{ }^{\circ} \mathrm{C}$. Crystals suitable for X-ray work were obtained by recrystallisation from ethanol. Anal. calcd. for $\mathrm{C}_{26} \mathrm{H}_{20} \mathrm{~F}_{2} \mathrm{~S}_{2}\left(434.56 \mathrm{~g} \mathrm{~mol}^{-1}\right): \mathrm{C}, 71.85 ; \mathrm{H}, 4.64$. Found $\mathrm{C}$, 71.77; $\mathrm{H}, 4.61 .{ }^{1} \mathrm{H}$ NMR: $\delta_{\mathrm{H}}\left(500.1 \mathrm{MHz}, \mathrm{CDCl}_{3}\right) 7.35\left(2 \mathrm{H}, \mathrm{dd},{ }^{3} J_{\mathrm{HH}} 7.8,{ }^{4} J_{H H} 1.0 \mathrm{~Hz}, \mathrm{H}-2\right), 7.29(2 \mathrm{H}$, pseudo-td, $\left.{ }^{3} \int_{\mathrm{HH}} 7.6,{ }^{4} J_{\mathrm{HH}} 1.5 \mathrm{~Hz}, \mathrm{H}-3\right), 7.22\left(2 \mathrm{H}\right.$, pseudo-td, $\left.{ }^{3} J_{\mathrm{HH}} 7.4,{ }^{4} \int_{\mathrm{HH}} 1.3 \mathrm{~Hz}, \mathrm{H}-4\right), 7.14-7.11(4 \mathrm{H}, \mathrm{m}$, $\mathrm{H}-9), 7.06\left(4 \mathrm{H}, \mathrm{dd},{ }^{3} J_{H H} 7.9,{ }^{4} J_{H H} 1.4 \mathrm{~Hz}, \mathrm{H}-5\right), 6.91-6.86(4 \mathrm{H}, \mathrm{m}, \mathrm{H}-10), 3.93\left(4 \mathrm{H}, \mathrm{ABq}, \Delta \delta_{A B} 0.02,{ }^{2} J_{H H}\right.$ $12.9 \mathrm{~Hz}, \mathrm{H}-7) .{ }^{13} \mathrm{C} N M R: \delta_{\mathrm{c}}\left(125.8 \mathrm{MHz}, \mathrm{CDCl}_{3}\right) 161.9\left(\mathrm{ArC}_{\mathrm{q}}, \mathrm{d},{ }^{1} J_{\mathrm{CF}} 245.6 \mathrm{~Hz}, \mathrm{C}-6\right), 141.2\left(\mathrm{ArC}_{\mathrm{q}}, \mathrm{C}-6\right)$, 135.7 ( $\left.\mathrm{ArC}_{q}, \mathrm{C}-1\right), 132.9\left(\mathrm{ArC}_{q}, \mathrm{~d},{ }^{4} \mathrm{~J}_{\mathrm{CF}} 3.2 \mathrm{~Hz}, \mathrm{C}-8\right), 130.5(\mathrm{ArCH}, \mathrm{C}-5), 130.4(\mathrm{ArCH}, \mathrm{C}-9), 129.3$ ( $\mathrm{ArCH}$, C-2), 128.5 (ArCH C-3), 126.1 (ArCH, C-4), $115.3\left(\mathrm{ArCH}, \mathrm{d},{ }^{2} \mathrm{~J}_{\mathrm{CF}} 21.7 \mathrm{~Hz}, \mathrm{C}-10\right), 37.8\left(\mathrm{CH}_{2}, \mathrm{C}-7\right) .{ }^{19} \mathrm{~F}\left\{{ }^{1} \mathrm{H}\right\}$ NMR: $\delta_{F}\left(376.5 \mathrm{MHz}, \mathrm{CDCl}_{3}\right)-115.55$ (s). HRMS (APCl+): $\mathrm{m} / z$ (\%) 184.0338 (45) $\left[\mathrm{M}-\mathrm{C}_{14} \mathrm{H}_{12} \mathrm{~F}_{2} \mathrm{~S}\right]^{+}$, $109.0444(100)\left[\mathrm{M}-\mathrm{C}_{19} \mathrm{H}_{14} \mathrm{FS}_{2}\right]^{+}$. IR (KBr): $\mathrm{v}_{\max } / \mathrm{cm}^{-1} 3060 \mathrm{w}\left(\mathrm{v}_{\text {Ar-H}}\right), 1597 \mathrm{~m}, 1504 \mathrm{~s}, 1214 \mathrm{~s}, 1087 \mathrm{~m}, 839 \mathrm{~s}$ $\left(v_{C-F}\right), 741 m\left(v_{C-s}\right)$. Raman (glass capillary): $v_{\max } / \mathrm{cm}^{-1} 3075 \mathrm{~s}\left(v_{\text {Ar-H }}\right), 1591 \mathrm{~s}, 1236 \mathrm{~m}, 1040 \mathrm{~m}, 851 \mathrm{~s}, 749 \mathrm{~s}$ $\left(v_{C-F}\right), 665 s\left(v_{C-s}\right)$. 


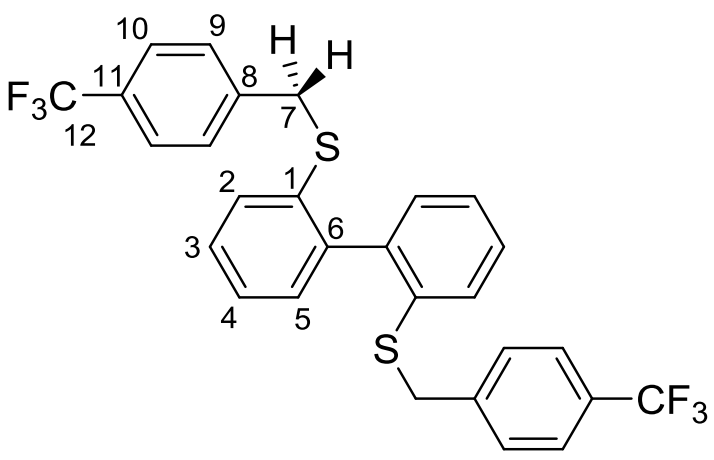

NMR atom numbering for 8 .

Prepared following method A using 3 (106 mg, $0.49 \mathrm{mmol}$ ), sodium hydroxide (42 mg, $1.08 \mathrm{mmol}$ ) and 1-(bromomethyl)-4-(trifluoromethyl)benzene ( $255 \mathrm{mg}, 1.07 \mathrm{mmol}$ ). Compound 8 was obtained as a crystalline solid (98 mg, $0.18 \mathrm{mmol}, 38 \%$ ), m.p 100-102 ${ }^{\circ} \mathrm{C}$. Crystals suitable for X-ray work were obtained by recrystallisation from ethanol. Anal. calcd. for $\mathrm{C}_{28} \mathrm{H}_{20} \mathrm{~F}_{6} \mathrm{~S}_{2}\left(534.58 \mathrm{~g} \mathrm{~mol}^{-1}\right): \mathrm{C}, 62.90 ; \mathrm{H}$, 3.77. Found $\mathrm{C}, 62.94 ; \mathrm{H}, 3.72 .{ }^{1} \mathrm{H}$ NMR: $\delta_{\mathrm{H}}\left(400.1 \mathrm{MHz}, \mathrm{CDCl}_{3}\right) 7.44\left(4 \mathrm{H}, \mathrm{d},{ }^{3} \mathrm{~J}_{\mathrm{HH}} 8.1 \mathrm{~Hz}, \mathrm{H}-10\right), 7.37(2 \mathrm{H}$, dd, $\left.{ }^{3} J_{\mathrm{HH}} 7.8,{ }^{4} J_{\mathrm{HH}} 1.0 \mathrm{~Hz}, \mathrm{H}-2\right), 7.33\left(2 \mathrm{H}\right.$, pseudo-td, $\left.{ }^{3} \mathrm{~J}_{\mathrm{HH}} 7.6,{ }^{4} \mathrm{~J}_{\mathrm{HH}} 1.5 \mathrm{~Hz}, \mathrm{H}-3\right), 7.23-7.20(6 \mathrm{H}, \mathrm{m}, \mathrm{H}-4,9)$, $6.98\left(2 \mathrm{H}, \mathrm{dd},{ }^{3} J_{\mathrm{HH}} 7.5,{ }^{4} J_{\mathrm{HH}} 1.4 \mathrm{~Hz}, \mathrm{H}-5\right), 3.95\left(4 \mathrm{H}, \mathrm{ABq}, \Delta \delta_{\mathrm{AB}} 0.01,{ }^{2} J_{\mathrm{HH}} 13.4 \mathrm{~Hz}, \mathrm{H}-7\right) .{ }^{13} \mathrm{C} N M R: \delta_{\mathrm{c}}(100.6$

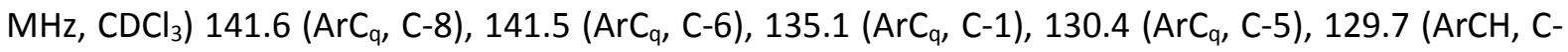
2), $129.3\left(\mathrm{ArC}_{\mathrm{q}}, \mathrm{q},{ }^{2} \mathrm{~J}_{\mathrm{CF}} 32.0 \mathrm{~Hz}, \mathrm{C}-11\right), 129.1$ (ArCH, C-9), $128.4(\mathrm{ArCH}, \mathrm{C}-3), 126.4(\mathrm{ArCH}, \mathrm{C}-4), 125.3$ $\left(\mathrm{ArCH}, \mathrm{q},{ }^{3} \mathrm{JF}_{\mathrm{CF}} 3.7 \mathrm{~Hz}, \mathrm{C}-10\right), 124.1\left(\mathrm{ArC}_{\mathrm{q}}, \mathrm{q},{ }^{1} \mathrm{~J}_{\mathrm{CF}} 273 \mathrm{~Hz}, \mathrm{C}-12\right), 38.3\left(\mathrm{CH}_{2}, \mathrm{C}-7\right) .{ }^{19} \mathrm{~F}\left\{{ }^{1} \mathrm{H}\right\} \mathrm{NMR}: \delta_{\mathrm{F}}(376.5$ $\left.\mathrm{MHz}, \mathrm{CDCl}_{3}\right)-62.52$ (s). HRMS (APCl+): $\mathrm{m} / z$ (\%) 535.0970 (5) $[\mathrm{M}+\mathrm{H}]^{+}, 159.0410$ (100) [M- $\left.\mathrm{C}_{20} \mathrm{H}_{14} \mathrm{~F}_{3} \mathrm{~S}_{2}\right]^{+}$. IR (KBr): $v_{\max } / \mathrm{cm}^{-1} 3056 \mathrm{w}\left(\mathrm{v}_{\mathrm{Ar}-\mathrm{H}}\right), 1616 \mathrm{~m}, 1455 \mathrm{~m}, 1420 \mathrm{~m}, 1323 \mathrm{~s}, 1121 \mathrm{~m}, 854 \mathrm{~m}\left(\mathrm{v}_{\mathrm{C}-\mathrm{F}}\right), 754 \mathrm{~m}\left(\mathrm{v}_{\mathrm{C}-\mathrm{s}}\right)$. Raman (glass capillary): $v_{\max } / \mathrm{cm}^{-1} 3063 \mathrm{w}\left(\mathrm{v}_{\mathrm{Ar}-\mathrm{H}}\right), 2917 \mathrm{~m}\left(\mathrm{v}_{\mathrm{C}-\mathrm{H}}\right), 1618 \mathrm{~m}, 1590 \mathrm{~s}, 1242 \mathrm{~s}, 1324 \mathrm{~m}\left(\mathrm{v}_{\mathrm{C}-\mathrm{F}}\right)$, $1044 \mathrm{~m}, 831 \mathrm{~m}, 713 \mathrm{~m}\left(\mathrm{v}_{\mathrm{c}-\mathrm{s}}\right)$. 


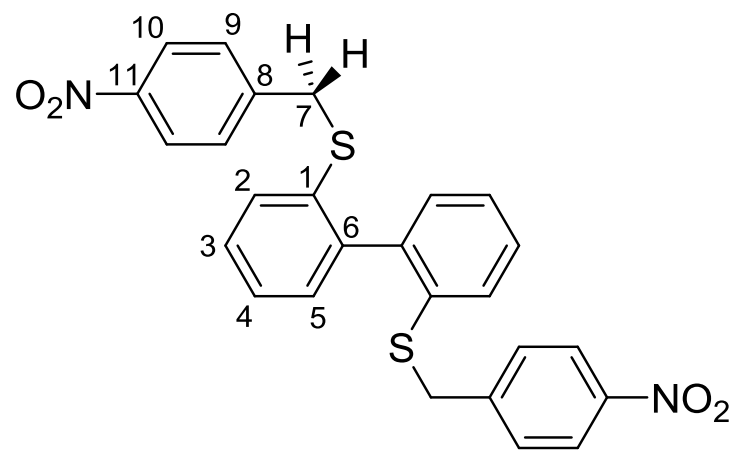

NMR atom numbering for 9 .

To a solution of 3 (106 mg, $0.49 \mathrm{mmol}$ ) dissolved in ethanol $(15 \mathrm{~mL})$, sodium hydroxide (42 $\mathrm{mg}, 1.08$ $\mathrm{mmol}$ ) was added and the solution was stirred for 30 minutes. 4-nitrobenzyl bromide (232 $\mathrm{mg}, 1.08$ mmol) was added to the reaction flask, and the mixture was stirred for 24 hours at room temperature. The solvent was removed and the crude product dissolved in dichloromethane (20 $\mathrm{mL}$ ). The reaction mixture was filtered under vacuum to remove the salt. The filtrate was concentrated under reduced pressure, yielding the crude product. Recrystallisation via a diffusion of diethyl ether into a saturated DCM solution containing the product resulted in colourless crystals (76 $\mathrm{mg}, 0.15 \mathrm{mmol}, 32 \%)$, m.p $185-188^{\circ} \mathrm{C}$. Crystal suitable for X-ray work were obtained by diffusion of diethyl ether into a saturated DCM solution of 9. Anal. calcd. for $\mathrm{C}_{26} \mathrm{H}_{20} \mathrm{~N}_{2} \mathrm{O}_{4} \mathrm{~S}_{2}\left(512.60 \mathrm{~g} \mathrm{~mol}^{-1}\right)$ : C, 63.91; H, 4.12; N, 5.73. Found C, 63.88; H, 3.96; N, 5.81. ${ }^{1} \mathrm{H}$ NMR: $\delta_{H}\left(500.1 \mathrm{MHz}, \mathrm{CDCl}_{3}\right) 8.06(4 \mathrm{H}, \mathrm{d}$, $\left.{ }^{3} J_{\mathrm{HH}} 8.7 \mathrm{~Hz}, \mathrm{H}-10\right), 7.38\left(2 \mathrm{H}, \mathrm{d},{ }^{3} \mathrm{JHH}_{\mathrm{HH}} 7.8 \mathrm{~Hz}, \mathrm{H}-2\right), 7.33\left(2 \mathrm{H}\right.$, pseudo-td, $\left.{ }^{3} \mathrm{~J}_{\mathrm{HH}} 7.6,{ }^{4} \mathrm{~J}_{\mathrm{HH}} 1.4 \mathrm{~Hz}, \mathrm{H}-3\right), 7.29-$ $7.23(6 \mathrm{H}, \mathrm{m}, \mathrm{H}-4,9), 7.02\left(2 \mathrm{H}, \mathrm{dd},{ }^{3} J_{\mathrm{HH}} 7.5,{ }^{4} J_{\mathrm{HH}} 1.2 \mathrm{~Hz}, \mathrm{H}-5\right), 3.97\left(4 \mathrm{H}, \mathrm{ABq}, \Delta \delta_{\mathrm{AB}} 0.02,{ }^{2} J_{H H} 13.6 \mathrm{~Hz}, \mathrm{H}-\right.$ 7). ${ }^{13} \mathrm{C}$ NMR: $\delta_{c}\left(125.8 \mathrm{MHz}, \mathrm{CDCl}_{3}\right) 146.9\left(\mathrm{ArC}_{\mathrm{q}}, \mathrm{C}-8\right), 145.2\left(\mathrm{ArC}_{\mathrm{q}}, \mathrm{C}-11\right), 141.9\left(\operatorname{ArC}_{\mathrm{q}}, \mathrm{C}-6\right), 134.4$ $\left(\mathrm{ArC}_{q}, \mathrm{C}-1\right), 130.4(\mathrm{ArCH}, \mathrm{C}-5), 130.3(\mathrm{ArCH}, \mathrm{C}-2), 129.6$ (ArCH, C-4), $128.6(\mathrm{ArCH}, \mathrm{C}-3), 126.9$ (ArCH, C9), 123.6 (ArCH, C-10), 38.3 ( $\left.\mathrm{CH}_{2}, \mathrm{C}-7\right)$. HRMS (APCl+): $\mathrm{m} / \mathrm{z}$ (\%) 216.0063 (100) $\left[\mathrm{M}-\mathrm{C}_{14} \mathrm{H}_{12} \mathrm{~N}_{2} \mathrm{O}_{4}\right]^{+}$, $184.0341(45)\left[\mathrm{M}-\mathrm{C}_{14} \mathrm{H}_{12} \mathrm{~N}_{2} \mathrm{O}_{4} \mathrm{~S}\right]^{+} . \mathrm{IR}(\mathrm{KBr}): \mathrm{v}_{\max } / \mathrm{cm}^{-1} 3072 \mathrm{w}\left(\mathrm{v}_{\mathrm{Ar}-\mathrm{H}}\right), 1601 \mathrm{~m}, 1517 \mathrm{~s}\left(\mathrm{v}_{\mathrm{N}-\mathrm{O}}\right), 1341 \mathrm{~s}\left(\mathrm{v}_{\mathrm{N}-\mathrm{O}}\right)$, $1087 \mathrm{~m}, 860 \mathrm{~s}, 754 \mathrm{~m}\left(\mathrm{v}_{\mathrm{C}-\mathrm{s}}\right)$. Raman (glass capillary): $\mathrm{v}_{\max } / \mathrm{cm}^{-1} 3070 \mathrm{w}\left(\mathrm{v}_{\mathrm{Ar}-\mathrm{H}}\right), 1601 \mathrm{~m}, 1520 \mathrm{~m}\left(\mathrm{v}_{\mathrm{N}-\mathrm{O}}\right)$, $1345 \mathrm{~s}\left(\mathrm{v}_{\mathrm{N}-\mathrm{O}}\right), 1110 \mathrm{~m}, 863 \mathrm{~m}, 754 \mathrm{~m}\left(\mathrm{v}_{\mathrm{c}-\mathrm{s}}\right)$. 


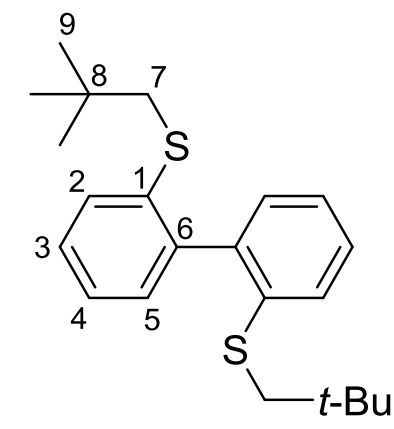

NMR atom numbering for 10.

To a solution of $3(101 \mathrm{mg}, 0.46 \mathrm{mmol})$ dissolved in ethanol $(15 \mathrm{~mL})$, sodium hydroxide (49 $\mathrm{mg}, 1.22$ $\mathrm{mmol})$ was added and the solution was stirred for 30 minutes. Neopentyl bromide $(0.13 \mathrm{~mL}, 1.03$ mmol) was added to the reaction flask and the mixture was stirred for 10 minutes at room temperature before being heated under reflux for 66 hours. The solvent was removed and the crude product dissolved in diethyl ether $(20 \mathrm{~mL})$. The reaction mixture was filtered under vacuum to remove the salt. The filtrate was concentrated under reduced pressure, yielding a white/pale yellow oil which slowly solidified over several days (114 mg, $0.32 \mathrm{mmol}, 70 \%) .{ }^{1} \mathrm{H}$ NMR: $\delta_{H}(500.1 \mathrm{MHz}$, $\left.\mathrm{CDCl}_{3}\right) 7.45(2 \mathrm{H}, \mathrm{dd}, J$ 8.0, $1.2 \mathrm{~Hz}, \mathrm{H}-2), 7.31\left(2 \mathrm{H}\right.$, pseudo-td, $\left.{ }^{3} J_{\mathrm{HH}} 7.6,{ }^{4} J_{\mathrm{HH}} 1.7 \mathrm{~Hz}, \mathrm{H}-3\right), 7.21(2 \mathrm{H}$, pseudo-td, $\left.{ }^{3} \mathrm{~J}_{\mathrm{HH}} 7.4,{ }^{4} J_{\mathrm{HH}} 1.2 \mathrm{~Hz}, \mathrm{H}-4\right), 7.16\left(2 \mathrm{H}, \mathrm{dd},{ }^{3} J_{\mathrm{HH}} 7.6,{ }^{4} \mathrm{~J}_{\mathrm{HH}} 1.7 \mathrm{~Hz}, \mathrm{H}-5\right), 2.75(4 \mathrm{H}, \mathrm{s}, \mathrm{H}-7), 0.92$ (18H, s, H-9). ${ }^{13} \mathrm{C}$ NMR: $\delta_{\mathrm{c}}\left(125.8 \mathrm{MHz}, \mathrm{CDCl}_{3}\right) 141.1$ ( $\left.\mathrm{ArC}_{\mathrm{q}}, \mathrm{C}-6\right), 137.7$ ( $\left.\mathrm{ArC}_{\mathrm{q}}, \mathrm{C}-1\right), 130.3$ (ArCH, C-5), 128.3 ( $\mathrm{ArCH}, \mathrm{C}-2), 127.9(\mathrm{ArCH}, \mathrm{C}-3), 124.9(\mathrm{ArCH}, \mathrm{C}-4), 48.1\left(\mathrm{CH}_{2}, \mathrm{C}-7\right), 32.18(\mathrm{C}, \mathrm{C}-8) 28.9\left(\mathrm{CH}_{3}, \mathrm{C}-\right.$ 9). HRMS (APCl+): $m / z(\%) 359.1864(100)[\mathrm{M}+\mathrm{H}]^{+}, 233.0100(25) . \mathrm{IR}(\mathrm{KBr}): v_{\max } / \mathrm{cm}^{-1} 3052 \mathrm{w}\left(\mathrm{v}_{\mathrm{Ar}-\mathrm{H}}\right)$, $2956 \mathrm{~s}\left(\mathrm{v}_{\mathrm{C}-\mathrm{H}}\right), 2865 \mathrm{~m}\left(\mathrm{v}_{\mathrm{C}-\mathrm{H}}\right), 1580 \mathrm{~m}, 1364 \mathrm{~m}, 1452 \mathrm{~s}, 1086 \mathrm{~s}, 751 \mathrm{~s}, 475 \mathrm{w}$.

\subsection{X-ray Crystallography}

Table 1 lists the details of data collections and refinements for 4-9. Data for 4-7 and $\mathbf{9}$ were collected using a Rigaku FRX (Mo-Ka, confocal optic) equipped with a Dectris P200 detector at $-100{ }^{\circ} \mathrm{C}$; for 8 using a Rigaku Saturn $724(\mathrm{Mo}-\mathrm{K} \alpha)$ at $-148{ }^{\circ} \mathrm{C}$. The data for all compounds were collected and processed using CrystalClear (Rigaku). The crystal structures were solved using direct methods and refined by full-matrix least-squares against $F^{2}$ (SHELXL) or heavy-atom Patterson methods and expanded using Fourier techniques.[28] The non-hydrogen atoms were refined anisotropically, hydrogen atoms were assigned riding isotropic displacement parameters and constrained to idealised geometries. Searches of the Cambridge Structure Database were performed using the WebCSD.[29]. These data can be obtained free of charge via 
www.ccdc.cam.ac.uk/conts/retrieving.html or from the Cambridge Crystallographic Data Centre, 12 Union Road, Cambridge CB2 1EZ, UK; fax (+44) 1223-336-033; e-mail: deposit@ccdc.cam.ac.uk. CCDC Numbers XXXXX-XXXXX.

Table 1: Details of the X-ray data collections and refinements for compounds 4-9.

\begin{tabular}{|c|c|c|c|c|c|c|}
\hline Compound & 4 & 5 & 6 & 7 & 8 & 9 \\
\hline Formula & $\mathrm{C}_{26} \mathrm{H}_{22} \mathrm{~S}_{2}$ & $\mathrm{C}_{28} \mathrm{H}_{26} \mathrm{~S}_{2}$ & $\mathrm{C}_{26} \mathrm{H}_{20} \mathrm{Br}_{2} \mathrm{~S}_{2}$ & $\mathrm{C}_{26} \mathrm{H}_{20} \mathrm{~F}_{2} \mathrm{~S}_{2}$ & $\mathrm{C}_{28} \mathrm{H}_{20} \mathrm{~F}_{6} \mathrm{~S}_{2}$ & $\mathrm{C}_{26} \mathrm{H}_{20} \mathrm{~N}_{2} \mathrm{O}_{4} \mathrm{~S}_{2}$ \\
\hline$M$ & 398.58 & 426.63 & 536.37 & 434.56 & 534.58 & 488.57 \\
\hline Crystal system & Triclinic & Monoclinic & Monoclinic & Triclinic & Monoclinic & Monoclinic \\
\hline Space group & $P-1$ & $12 / a$ & $P 2_{1} / n$ & $P-1$ & $P 21 / n$ & $P 2_{1} / n$ \\
\hline$a / \AA$ & $9.663(4)$ & $19.162(8)$ & $10.942(3)$ & $7.9777(18)$ & $11.318(8)$ & $8.0281(10)$ \\
\hline$b / \AA$ & $9.882(5)$ & $6.116(3)$ & $7.7060(19)$ & $11.591(2)$ & $7.664(5)$ & $36.034(5)$ \\
\hline$c / \AA$ & $11.617(6)$ & $20.836(4)$ & $26.893(7)$ & $12.626(2)$ & 27.051(19) & $15.957(2)$ \\
\hline$\alpha$ & $111.388(10)$ & 90 & 90 & $68.091(14)$ & 90 & 90 \\
\hline B & $93.614(6)$ & $112.12(2)$ & $91.039(5)$ & $75.561(15)$ & $93.152(17)$ & $102.219(3)$ \\
\hline$r$ & $91.962(11)$ & 90 & 90 & $81.733(17)$ & 90 & 90 \\
\hline$U / A^{3}$ & $1028.9(9)$ & $2265.6(15)$ & $2267.2(10)$ & $1047.3(4)$ & $2343(3)$ & $4511.5(10)$ \\
\hline$Z$ & 2 & 4 & 4 & 2 & 4 & 8 \\
\hline$\mu / m m^{-1}$ & 0.267 & 0.247 & 3.782 & 0.282 & 0.291 & 0.274 \\
\hline Reflections collected & 13941 & 9504 & 19488 & 15119 & 30073 & 44187 \\
\hline Independent reflections & 3703 & 2041 & 4157 & 3810 & 4300 & 8266 \\
\hline$R_{\text {int }}$ & 0.0541 & 0.0478 & 0.0299 & 0.0500 & 0.0606 & 0.0447 \\
\hline$R_{\text {all }}$ & 0.0639 & 0.0649 & 0.0390 & 0.0634 & 0.1039 & 0.0528 \\
\hline$w R 2[I>2 \sigma(I)]$ & 0.1439 & 0.1255 & 0.0637 & 0.1210 & 0.1797 & 0.1251 \\
\hline
\end{tabular}

\section{Results and Discussion}

\subsection{Synthesis}

The previous method of synthesising chalcogenoether compounds on naphthalene and acenaphthene used within the Woollins group started from 1,8-dibromonaphthalene and 5,6dibromoacenaphthene respectively. A low temperature lithium halogen exchange using $n$-butyl lithium followed by addition of RE-ER (where $\mathrm{R}=$ alkyl or aryl; $\mathrm{E}=\mathrm{S}, \mathrm{Se}, \mathrm{Te}$ ) resulted in the desired compounds. 2,2'-dibromo-1,1'-biphenyl is easily prepared in good yields $(74 \%)$ as shown in Scheme 1,[25] the addition of 2 molar equivalents of $n$-butyl lithium to 1 followed by PhE-EPh (E = S, Te) resulted in inseparable mixtures. Some evidence for the successful formation of the tellurium analogue was found within the mass spectrum with a signal at $\mathrm{m} / \mathrm{z} 562.9575$ with the isotope pattern matching that predicted for this $[\mathrm{M}+\mathrm{H}]^{+}$peak (Figures $\mathrm{S} 1$ and $\mathrm{S} 2$ in ESI). In the case of the 
tellurium derivative the main compound isolated after a purification attempt using column chromatography was dibenzo[b,d]tellurophene $(43 \%)$.

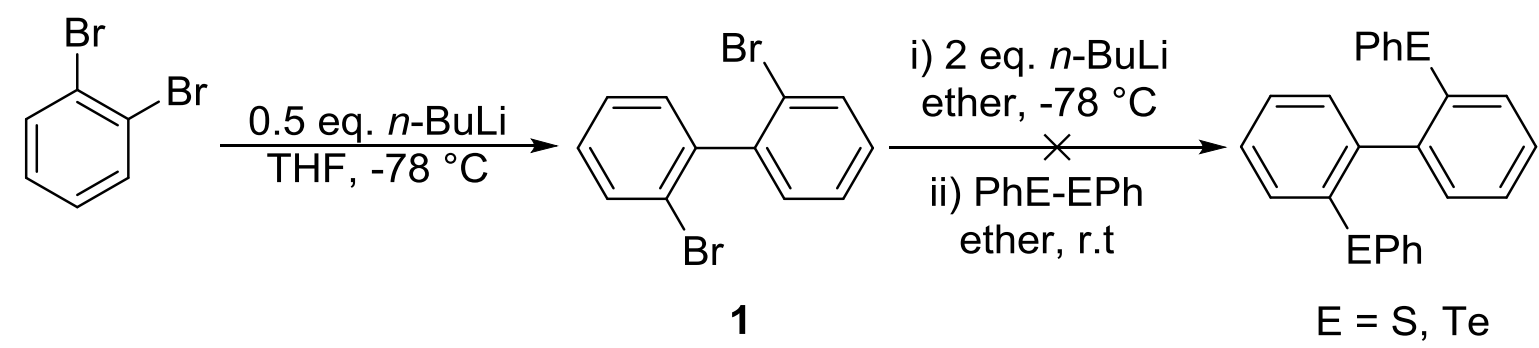

Scheme 1: Initial synthetic route towards bis(chalcogen) biphenyls.

Since adding the aryl-chalcogen species directly onto the biphenyl backbone was unsuccessful a new synthetic route starting from $\mathbf{2}$ was devised for the sulfur derivatives (Scheme 2). The equivalent tellurium compound is unknown in the literature with attempts to prepare it resulting in the formation of dibenzo[b,d]tellurophene.[30] For this reason the sulfur compounds became the focus for the rest of this investigation. By forming a reactive dithiolate species a simple $\mathrm{S}_{\mathrm{N}} 2$ reaction with a compound such as $\mathrm{R}-\mathrm{Br}$ was expected to occur. The reduction of $\mathbf{2}$ to $1,1^{\prime}$-biphenyl-2,2'-dithiol (3) using sodium borohydride was achieved almost quantitatively (yield >99\%). [26] The deprotonation of $\mathbf{3}$ to form the dithiolate is easily achieved using a base such as sodium hydroxide. We chose a series of benzyl bromides with varying substituents in the para position of the phenyl ring in addition to neopentyl bromide to react with the biphenyl dithiolate (Scheme 2). 


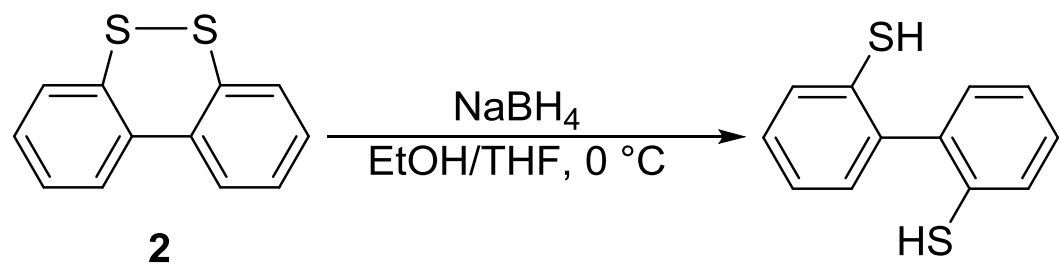

3

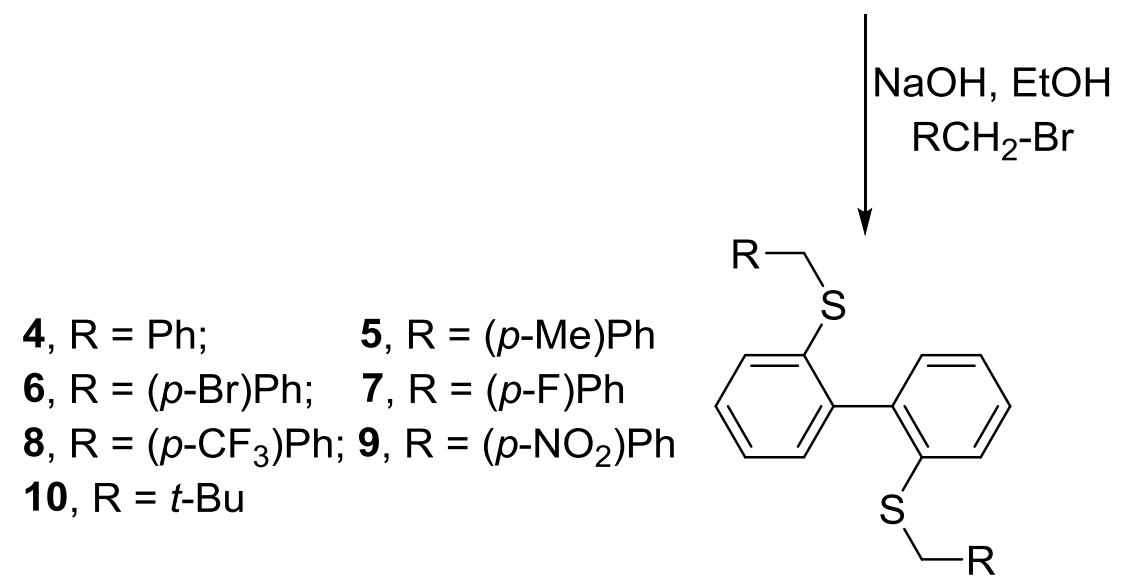

Scheme 2: Synthetic route to 2,2'-bis(alkylthio)-1,1'-biphenyls 4-10.

Compound 3 was stirred in ethanol with 2 equivalents of sodium hydroxide for $0.5 \mathrm{~h}$ prior to addition of the alkyl bromide. Compounds 4-9 were obtained in 32-69\% yields as white/off-white solids after purification. Purification of 4-8 was achieved via recrystallisation from boiling ethanol resulting in crystals which were suitable for single crystal X-ray diffraction. Compound $\mathbf{9}$ was purified by recrystallisation from a mixture of $\mathrm{CH}_{2} \mathrm{Cl}_{2} /$ diethyl ether. During the attempt to recrystallise $\mathbf{1 0}$ the compound decomposed, attempts to purify via column chromatography also proved unsuccessful. The impurity present within $\mathbf{1 0}$ was identified as $\mathbf{2}$ via examination of its ${ }^{1} \mathrm{H}$ NMR spectrum; this showed that 10 made up $82 \%$ of the mixture allowing the yield to be calculated as $70 \%$. The bulk purity of 4-9 was determined by elemental analysis. Compound 9 has been previously reported by Millar in 1971 as part of a study synthesising macrocyclic compounds with sulfur and oxygen within the ring.[12] Modern spectroscopic techniques allow for a more detailed analysis of its ${ }^{1} \mathrm{H}$ NMR spectrum with additional ${ }^{13} \mathrm{C}$ and infrared spectroscopic data reported alongside its mass spectrum.

\subsection{Crystallographic Analysis}

Crystal structures of 4, 5, 7 and 9 are shown Figure 4 with 6 and 8 in Figure 5. Selected structural parameters listed in Table 2. All the $\mathrm{C}-\mathrm{S}$ bond lengths are comparable to the average $\mathrm{C}_{\mathrm{Alk}}-\mathrm{S}$ and $\mathrm{C}_{\mathrm{Ar}}-\mathrm{S}$ bond lengths $\left(\mathrm{C}_{\mathrm{Alk}}-\mathrm{S}, 1.762 \AA\right.$ and $\mathrm{C}_{\mathrm{Ar}}-\mathrm{S}, 1.790 \AA$ ) determined from similar structures within the Cambridge Structural Database.[31] The C-S-C angles all confirm the expected non-linear geometry around the sulfur atom with little deviation across the series (C-S-C, 99.3(1)-104.6(1) ${ }^{\circ}$. The torsion 
angle between the two phenyl rings within the biphenyl backbone shows more variation. Compounds $5\left(72.1(3)^{\circ}\right), 6\left(-84.7(3)^{\circ}\right), 7\left(-76.7(3)^{\circ}\right)$ and $8\left(-83.9(6)^{\circ}\right)$ all have values below $90^{\circ}$ as the two phenyl rings close up slightly. Compounds $4\left(-112.3(3)^{\circ}\right)$ and $9\left(110.0(2)^{\circ}\right)$ both display torsion angles greater than $90^{\circ}$ as the phenyl rings move further apart.

Table 2: Selected bond lengths $[\AA ̊]$, angles $\left[{ }^{\circ}\right]$ and torsions $\left[{ }^{\circ}\right]$ for $4-9$.

\begin{tabular}{cccc}
\hline & $\mathbf{4}$ & $\mathbf{5}$ & $\mathbf{6}$ \\
\hline $\mathrm{S}-\mathrm{C}^{\dagger}$ & $1.765(3)-1.822(3)$ & $1.768(2)-1.822(3)$ & $1.775(3)-1.827(2)$ \\
$\mathrm{C} 1-\mathrm{S} 1-\mathrm{C} 13$ & $104.4(1)$ & - & $101.2(1)$ \\
$\mathrm{C} 1-\mathrm{S} 1-\mathrm{C} 7$ & - & $103.4(5)$ & - \\
$\mathrm{C} 8-\mathrm{S} 8-\mathrm{C} 20$ & $103.4(1)$ & - & $104.6(1)$ \\
$\mathrm{C} 1-\mathrm{C} 2-\mathrm{C} 7-\mathrm{C} 8$ & $-112.3(3)$ & - & $-84.7(3)$ \\
$\mathrm{C} 1-\mathrm{C} 2-\mathrm{C} 2^{\prime}-\mathrm{C} 1^{\prime}$ & - & $72.1(3)$ & - \\
\hline & $\mathbf{7}$ & $\mathbf{8}$ & $\mathbf{9}$ \\
\hline $\mathrm{S}-\mathrm{C}^{\dagger}$ & $1.773(2)-1.824(3)$ & $1.753(5)-1.815(5)$ & $1.779(2)-1.826(2)$ \\
$\mathrm{C} 1-\mathrm{S} 1-\mathrm{C} 13$ & $102.5(1)$ & $100.9(2)$ & $101.71(9)[102.32(8)]$ \\
$\mathrm{C} 8-\mathrm{S} 8-\mathrm{C} 20$ & $99.3(1)$ & - & $102.40(9)[101.92(9)]$ \\
$\mathrm{C} 8-\mathrm{S} 8-\mathrm{C} 21$ & - & $104.8(2)$ & - \\
$\mathrm{C} 1-\mathrm{C} 2-\mathrm{C} 7-\mathrm{C} 8$ & $-76.7(3)$ & $-83.9(6)$ & $110.0(2)[-110.4(2)]$ \\
\hline
\end{tabular}

[] denotes from second molecule within the asymmetric unit.

${ }^{+}$ranges quoted for $\mathrm{S}-\mathrm{C}$ bond lengths of molecules within the asymmetric unit. 

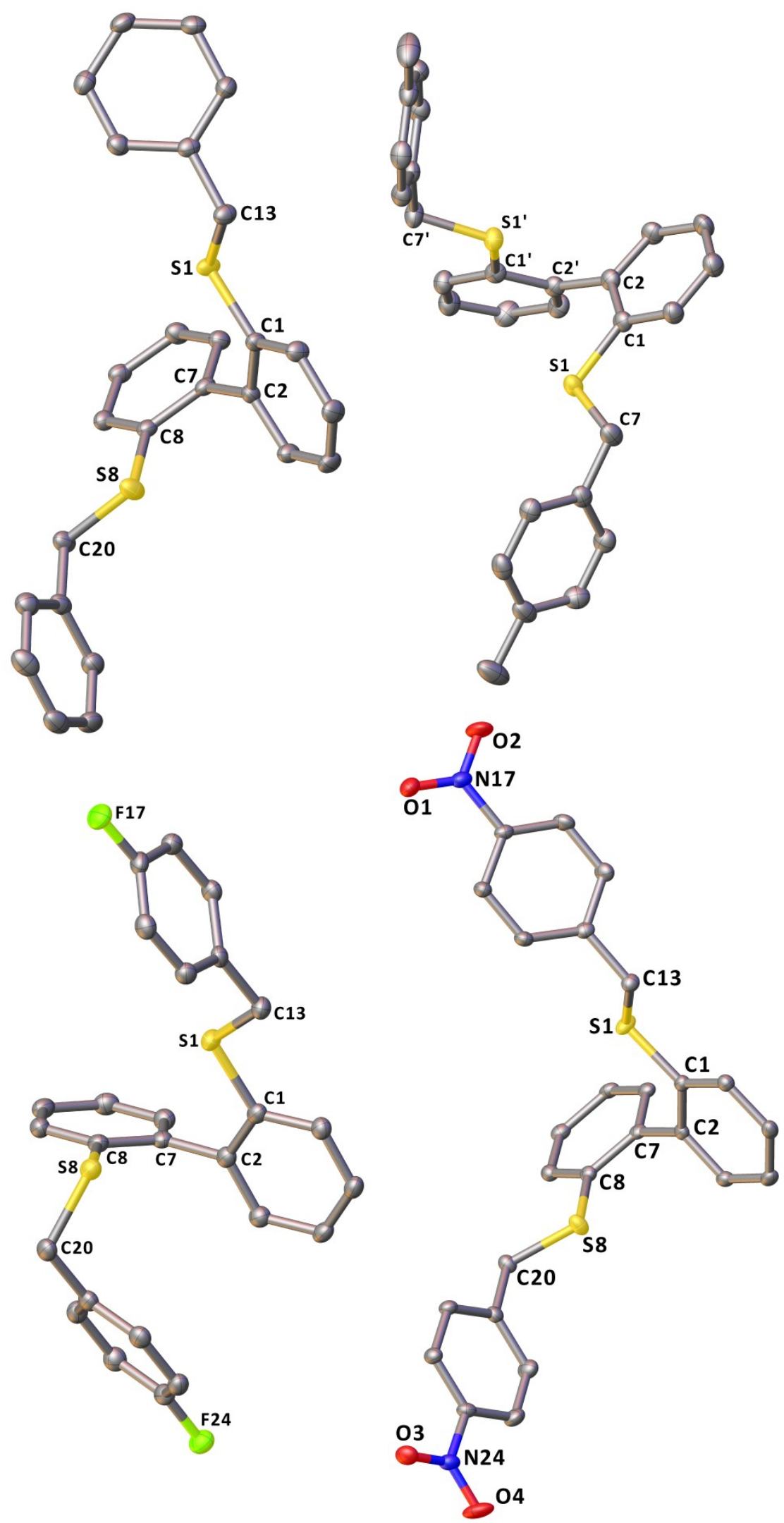

Figure 4: Crystal structures of $\mathbf{4}$ (top left), $\mathbf{5}$ (top right), $\mathbf{7}$ (bottom left) and $\mathbf{9}$ (bottom right). Only one molecule within the asymmetric unit is shown for $\mathbf{9}$. Hydrogen atoms omitted for clarity, ellipsoids plotted at the $40 \%$ probability level. 
Two different conformers are present within the crystal structures of compounds 4-9. For 4, 5, 7 and 9 the compound attains an "open" structure, i.e. the two benzyl substituents point away from each other (anti-parallel). This is expected as it results in minimal steric repulsion between the two benzyl groups. Interestingly in $\mathbf{6}$ and $\mathbf{8}$ the structures adopt a "closed" structure with the two benzyl substituents pointing roughly in the same direction (parallel). This is likely due to packing effects with long range $\mathrm{Br}-\mathrm{H}(3.044 \AA$ ) and $\mathrm{F}-\mathrm{H}(2.594 \AA)$ interactions present within 6 and 8 respectively.
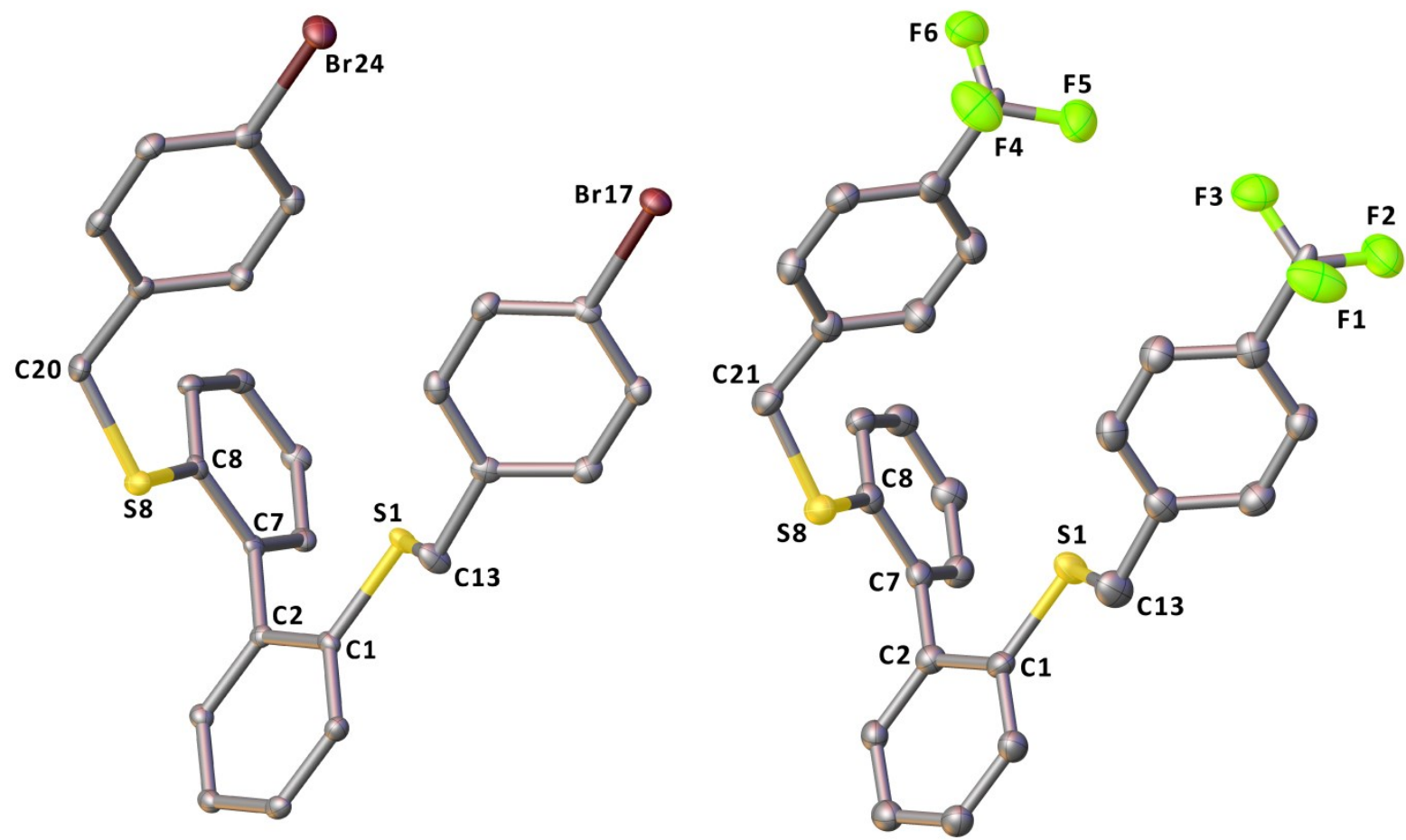

Figure 5: Crystal structures of 6 (left) and 8 (right). Hydrogen atoms omitted for clarity, ellipsoids plotted at the $40 \%$ probability level.

\subsection{Spectroscopic Analysis}

The benzyl and neopentyl bromide starting materials all display singlets in their ${ }^{1} \mathrm{H}$ NMR spectra corresponding to the $\mathrm{CH}_{2}$ fragment ( $\delta_{\mathrm{H}} 4.43-4.53$ (benzyls) and 3.27 (neopentyl) ppm). Compounds 4-10 all display an upfield shift in this signal with the benzyl derivatives at $\delta_{H} 3.93-3.99 \mathrm{ppm}$ and the neopentyl at $\delta_{\mathrm{H}} 2.75 \mathrm{ppm}$. This upfield shift is as a result of exchanging the bromine atom with a more donating sulfur atom. Interestingly in the case of 4-9 this signal within the ${ }^{1} \mathrm{H}$ NMR spectra appears as four lines in an $A B$ system. The signal appears this way as the difference in chemical shift between the two $\mathrm{CH}_{2}$ protons determined in hertz is very close in magnitude to the coupling between them. This second order coupling suggests restricted rotation around the aryl-aryl bond in the biphenyl backbone. This restricted rotation arises owing to the bulky nature of the benzyl groups 
in the ortho positions preventing free rotation around the biphenyl aryl-aryl bond. Such restriction results in axial chirality[32] within the molecule which causes the nearby hydrogen atoms $\left(\mathrm{CH}_{2}\right.$ group) to become diastereotopic. Similar observations were made by Mazzanti during their investigation into hindered biphenyl carbinols.[33] They used enantioselective HPLC to resolve the enantiomers and determined the absolute configuration on the basis of the obtained CD (circular dichroism) spectra. The true centres for each of the doublets within the AB systems for 4-9 were determined with the calculations included within the supplementary information. The true centres determined differed only slightly from those seen using the ${ }^{1} \mathrm{H}$ NMR spectra directly $\left(\Delta \delta_{A B} \approx 0.02\right.$ ppm). Figure 6 shows the $A B$ system present within 4 with the true centres of each doublet highlighted. For 10 this signal appears as a singlet indicating the aryl-aryl bond can now freely rotate. Within the aromatic region of 4-9 the signals corresponding to the phenyl ring of the benzyl group display shifts downfield compared to those in the respective starting materials. For the fluorine containing compound $\mathbf{7}$ these signals appeared as multiplets due to coupling to the NMR active fluorine atom.
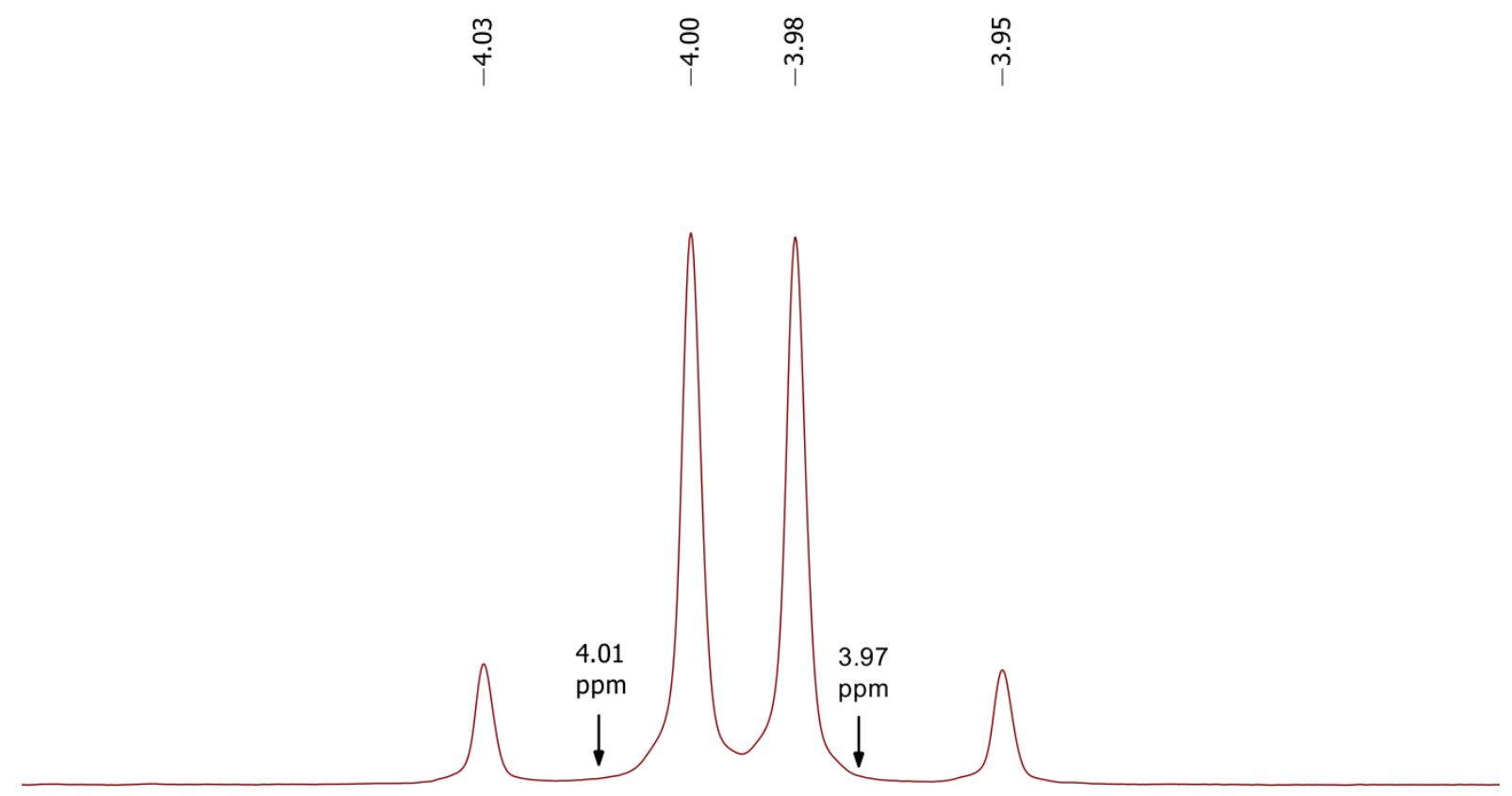

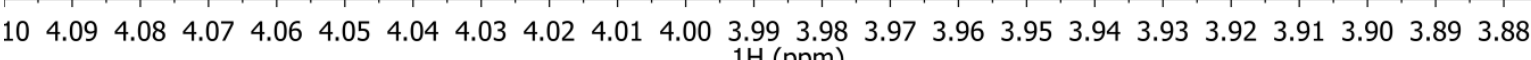
$1 \mathrm{H}(\mathrm{ppm})$

Figure 6: $A B$ system present within the ${ }^{1} \mathrm{H}$ NMR spectrum of $\mathbf{4}$ with the calculated true centres shown. 
The biphenyl backbone was expected to result in four signals, two doublet of doublets $\left(H_{a}\right.$ and $H_{d}$, Figure 7) and two doublet of doublet of doublets $\left(H_{b}\right.$ and $H_{c}$, Figure 7) in the ${ }^{1} H$ NMR spectra. However, the signals corresponding to $H_{b}$ and $H_{c}$ actually appear as pseudo triplet of doublets. Taking $H_{b}$ as an example this occurs due to the almost identical magnitude of the ${ }^{3} J_{H H}$ coupling between $H_{b}$ and $H_{a}$ as well as $H_{b}$ and $H_{c}$ as shown in Figure 7. These observations mirror those made in other compounds where we have used the biphenyl dithiolate backbone.[34, 35] The aromatic signals arising from the benzyl substituents in 4-9 are present as expected with some appearing as multiplets due to overlapping with the biphenyl signals.
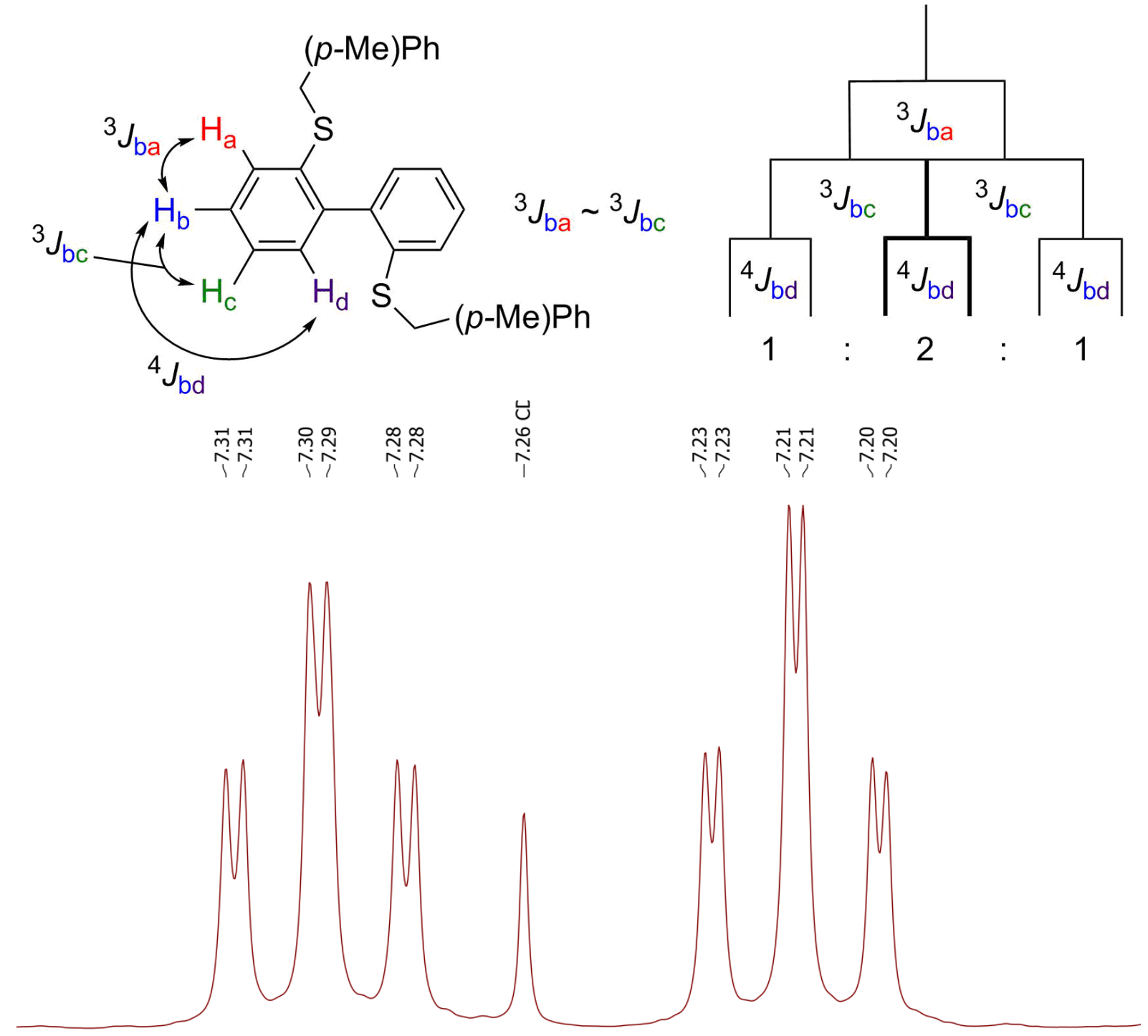

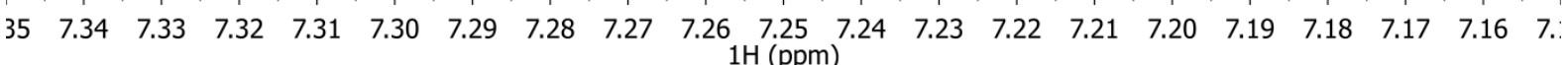

Figure 7: The section of the ${ }^{1} \mathrm{H}$ NMR spectrum of 5 showing the two pseudo triplet of doublets with a splitting diagram showing how these signals are formed. 
${ }^{13} \mathrm{C}$ NMR spectra were obtained for 4-10 and in all cases the number of signal corresponding to quaternary, $\mathrm{CH}$ and $\mathrm{CH}_{3}$ carbon atoms matched what was expected. No indication of any other species being present was observed for 4-9. The expected signals for the impurity (2) within the ${ }^{13} \mathrm{C}$ NMR spectrum of $\mathbf{1 0}$ were observed. The fluorine containing compounds $\mathbf{7}$ and $\mathbf{8}$ resulted in the phenyl ring carbon atoms of the benzyl group being split into doublets as expected. The $\mathrm{CF}_{3}$ group in 8 gave rise to a quartet in the ${ }^{13} \mathrm{C}$ NMR spectrum $\left(\delta_{\mathrm{C}} 124.1 \mathrm{ppm}\right)$ with a large ${ }^{1} J_{\mathrm{CF}}$ coupling constant of $273 \mathrm{~Hz}$ which was similar to other compounds incorporating the $\mathrm{S}-\mathrm{CH}_{2}-\left(\mathrm{Ph}\left(p-\mathrm{CF}_{3}\right)\right)$ moiety.[36] The ${ }^{19} \mathrm{~F}\left\{{ }^{1} \mathrm{H}\right\}$ NMR spectra of 7 and 8 showed only one signal at $\delta_{F}-115.6(7)$ and $-62.5(8)$ ppm providing further evidence that only one species is present. High resolution mass spectroscopy of 4-10 showed $[\mathrm{M}+\mathrm{H}]^{+}$ions for 4, 5, 8 and $\mathbf{1 0}$ each displaying the expected isotopic distribution pattern. For $\mathbf{6 , 7}$ and 9 signals corresponding to $\left[\mathrm{XPhCH}_{2}\right]^{+}\left(\mathrm{X}=\mathrm{Br}(6), \mathrm{F}(7), \mathrm{NO}_{2}(9)\right)$ and $[\mathrm{BiphenS}]^{+}$were observed however no $[\mathrm{M}+\mathrm{H}]^{+}$peaks were present.

\section{Conclusions}

A series of bis(alkyl)thioethers based upon a biphenyl backbone were successfully synthesised. The benzyl containing compounds all displayed an $\mathrm{AB}$ quartet for the benzyl $\mathrm{CH}_{2}$ signal owing to axially chirality introduced by restricted rotation around the aryl-aryl bond of the biphenyl backbone. When the benzyl group was changed to neopentyl this signal appeared as a singlet as the biphenyl backbone can now freely rotate. Analysis of the solid state structures of the benzyl derivatives showed two conformers, anti-parallel where the benzyl groups point away from each other and parallel where they are pointing in the same direction. The potential of these compounds to act as ligands will be investigated next. 
[1] J. Drabowicz, J. Lewkowski, W. Kudelska, T. Girek, Science of Synthesis, Thieme Chemistry2008.

[2] J.L. García Ruano, M.B. Cid, A.M. Martín-Castro, J. Alemán, Science of Synthesis, Thieme Chemistry2008.

[3] M. Kazemi, L. Shiri, H. Kohzadi, Recent Advances in Aryl Alkyl and Dialkyl Sulfide Synthesis, Phosphorus, Sulfur, and Silicon and the Related Elements 190(7) (2014) 978-1003.

[4] P.D. de Koning, L. Murtagh, J.P. Lawson, R.A. Vonder Embse, S.A. Kunda, W. Kong, Development an Efficient Route to the 5-Lipoxygenase Inhibitor PF-04191834, Organic Process Research \& Development 15(5) (2011) 1046-1051.

[5] L.R. Gahan, Cyclononanes: The extensive chemistry of fundamentally simple ligands, Coord. Chem. Rev. 311 (2016) 168-223.

[6] M. Kuriyama, Development of Efficient Catalytic Arylation of Aldehydes with ThioetherImidazolinium Carbene Ligands, Chem. Pharm. Bull. 60(4) (2012) 419-428.

[7] J.T. Manka, F. Guo, J. Huang, H. Yin, J.M. Farrar, M. Sienkowska, V. Benin, P. Kaszynski, Synthesis of Polyfunctionalized Biphenyls as Intermediates for a New Class of Liquid Crystals, J. Org. Chem. 68(25) (2003) 9574-9588.

[8] W.B. Price, S. Smiles, Nephthylene 1,8-disulfide, J. Chem. Soc. (Copyright (C) 2016 American Chemical Society (ACS). All Rights Reserved.) (1928) 2372-4.

[9] R. Ros, M. Vidali, R. Graziani, Synthesis of Group VIB carbonyl complexes with diarsine and disulfides. Molecular structure of W(CO)4[o-bis(methylthio)benzene], Gazz. Chim. Ital. 100(Copyright (C) 2016 American Chemical Society (ACS). All Rights Reserved.) (1970) 407-23.

[10] J. Meinwald, D. Dauplaise, F. Wudl, J.J. Hauser, Peri-bridged naphthalenes from 1,8dilithionaphthalene, J. Am. Chem. Soc. 99(Copyright (C) 2016 American Chemical Society (ACS). All Rights Reserved.) (1977) 255-7.

[11] H. Bock, G. Braehler, Radical ions. XXXIII. Oxidation and reduction of methylthio-substituted naphthalenes - a comparison of molecular states, Chem. Ber. 112(Copyright (C) 2016 American Chemical Society (ACS). All Rights Reserved.) (1979) 3081-97.

[12] D.W. Allen, P.N. Braunton, I.T. Millar, J.C. Tebby, Synthesis of novel macrocyclic compounds containing sulphur or oxygen in the ring: thia- and oxa-cyclophanes. Mass spectral and other studies, Journal of the Chemical Society C: Organic (0) (1971) 3454-3466.

[13] M. Barbero, S. Bazzi, S. Cadamuro, S. Dughera, C. Magistris, P. Venturello, A new effective synthesis of arene mono- and disulfonyl chlorides, Synlett (Copyright (C) 2016 American Chemical Society (ACS). All Rights Reserved.) (2010) 1803-1806.

[14] F. Di Furia, G. Licini, G. Modena, O. De Lucchi, Asymmetric oxidation of thio ethers. Part 4. Optical resolution of 1,1'-binaphthalene-2,2'-dithiol, Tetrahedron Lett. 30(Copyright $($ (C) 2016 American Chemical Society (ACS). All Rights Reserved.) (1989) 2575-6.

[15] F.R. Knight, A.L. Fuller, M. Buhl, A.M. Slawin, J.D. Woollins, Synthetic and structural studies of 1,8-chalcogen naphthalene derivatives, Chem. Eur. J. 16(25) (2010) 7503-16.

[16] H. Shima, N. Furukawa, Rearrangements of the allyl group in the thermolysis of 1,8-bis(allylthio)and 1,8-bis(allylseleno)naphthalene monooxides via through-space interaction between two sulfur and selenium atoms, Tetrahedron 51(Copyright (C) 2016 American Chemical Society (ACS). All Rights Reserved.) (1995) 12239-56.

[17] L.K. Aschenbach, F.R. Knight, R.A.M. Randall, D.B. Cordes, A. Baggott, M. Buehl, A.M.Z. Slawin, J.D. Woollins, Onset of three-center, four-electron bonding in peri-substituted acenaphthenes: $A$ structural and computational investigation, Dalton Trans. 41(Copyright (C) 2016 American Chemical Society (ACS). All Rights Reserved.) (2012) 3141-3153.

[18] F. Sibbel, C.G. Daniliuc, A. Studer, 2,2'-Bis-substituted Biphenyls by the Addition of Nucleophiles to Benzyne Followed by In Situ Oxidative Homocoupling, Eur. J. Org. Chem. 2015(21) (2015) 46354644.

[19] R. Mariz, A. Poater, M. Gatti, E. Drinkel, J.J. Bürgi, X. Luan, S. Blumentritt, A. Linden, L. Cavallo, R. Dorta, C2-Symmetric Chiral Disulfoxide Ligands in Rhodium-Catalyzed 1,4-Addition: From Ligand Synthesis to the Enantioselection Pathway, Chem. Eur. J. 16(48) (2010) 14335-14347. 
[20] F.R. Knight, R.A.M. Randall, T.L. Roemmele, R.T. Boeré, B.E. Bode, L. Crawford, M. Bühl, A.M.Z. Slawin, J.D. Woollins, Electrochemically Informed Synthesis: Oxidation versus Coordination of 5,6Bis(phenylchalcogeno)acenaphthenes, ChemPhysChem 14(14) (2013) 3199-3203.

[21] L.M. Diamond, F.R. Knight, K.S. Athukorala Arachchige, R.A.M. Randall, M. Bühl, A.M.Z. Slawin, J.D. Woollins, Bridging the Gap: Attractive 3c-4e Interactions in peri-Substituted Acenaphthylenes, Eur. J. Inorg. Chem. 2014(9) (2014) 1512-1523.

[22] A. Nordheider, E. Hupf, B.A. Chalmers, F.R. Knight, M. Bühl, S. Mebs, L. Chęcińska, E. Lork, P.S. Camacho, S.E. Ashbrook, K.S. Athukorala Arachchige, D.B. Cordes, A.M.Z. Slawin, J. Beckmann, J.D. Woollins, Peri-Substituted Phosphorus-Tellurium Systems-An Experimental and Theoretical Investigation of the P...Te through-Space Interaction, Inorg. Chem. 54(5) (2015) 2435-2446.

[23] Y. Imai, W. Zhang, T. Kida, Y. Nakatsuji, I. Ikeda, Novel Axial Chiral Sulfur-oxazoline Ligands with a Biphenyl Backbone, Synlett 1999(08) (1999) 1319-1321.

[24] W.L.F. Armarego, C.L.L. Chai, Purification of Laboratory Chemicals, 6th ed., ButterworthHeinemann, Oxford, 2009.

[25] H. Gilman, B.J. Gaj, Coupling reactions with some organolithium compounds in tetrahydrofuran, J. Org. Chem. 22(Copyright (C) 2016 American Chemical Society (ACS). All Rights Reserved.) (1957) 447-9.

[26] K. Yui, Y. Aso, T. Otsubo, F. Ogura, Syntheses and Properties of 2,2'-Binaphtho[1,8-De]-1,3Dithiinylidene and Its Selenium Analog, 2-(1,3-Dithiol-2-Ylidene)Naphtho[1,8-De]-1,3-Dithiin, and 2(4h-Thiopyran-4-Ylidene)Naphtho[1,8-De]-1,3-Dithiin, Bull. Chem. Soc. Jpn. 61(3) (1988) 953-959.

[27] A.J. Ashe, J.W. Kampf, P.M. Savla, The reaction of sulfur with dilithio compounds. The syntheses and structures of phenanthro [1, 10-cd]-1,2-dithiole and phenanthro[4,5-cde] [1,2]dithiin, Heteroat. Chem 5(2) (1994) 113-119.

[28] G. Sheldrick, A short history of SHELX, Acta Crystallogr. A 64(1) (2008) 112-122.

[29] I.R. Thomas, I.J. Bruno, J.C. Cole, C.F. Macrae, E. Pidcock, P.A. Wood, WebCSD: the online portal to the Cambridge Structural Database, J. Appl. Crystallogr. 43(2) (2010) 362-366.

[30] S. Murata, T. Suzuki, A. Yanagisawa, S. Suga, Syntheses of dibenzo[c,e][1,2]diselenin and related novel chalcogenide heterocyclic compounds, J. Heterocycl. Chem. 28(2) (1991) 433-438.

[31] C.F. Macrae, I.J. Bruno, J.A. Chisholm, P.R. Edgington, P. McCabe, E. Pidcock, L. RodriguezMonge, R. Taylor, J. van de Streek, P.A. Wood, Mercury CSD 2.0 - new features for the visualization and investigation of crystal structures, J. Appl. Crystallogr. 41(2) (2008) 466-470.

[32] F. Leroux, Atropisomerism, Biphenyls, and Fluorine: A Comparison of Rotational Barriers and Twist Angles, ChemBioChem 5(5) (2004) 644-649.

[33] D. Casarini, L. Lunazzi, M. Mancinelli, A. Mazzanti, C. Rosini, Structure, Conformation, Stereodynamics, Dimer Formation, and Absolute Configuration of Axially Chiral Atropisomers of Hindered Biphenyl Carbinols, J. Org. Chem. 72(20) (2007) 7667-7676.

[34] P.S. Nejman, B. Morton-Fernandez, D.J. Moulding, K.S. Athukorala Arachchige, D.B. Cordes, A.M.Z. Slawin, P. Kilian, J.D. Woollins, Structural diversity of bimetallic rhodium and iridium half sandwich dithiolato complexes, Dalton Trans. 44(38) (2015) 16758-16766.

[35] C.B.E. Meigh, P.S. Nejman, A.M.Z. Slawin, J. Derek Woollins, Complexation of aromatic dichalcogen ligands to germanium, Inorg. Chim. Acta 456 (2017) 120-127.

[36] S.L. Buchwald, R.B. Nielsen, Kinetics and substituent effects in the formation of zirconocene thioaldehyde complexes: $\beta$-hydride elimination versus cyclometalation, J. Am. Chem. Soc. 110(Copyright (C) 2016 American Chemical Society (ACS). All Rights Reserved.) (1988) 3171-5. 